\title{
The Application of Stability Bias in Conceptual Learning
}

\author{
Ziyi Liu ${ }^{1}$ \\ ${ }^{1}$ Viewpoint School, 23620 Mulholland Highway, Calabasas, California 91302, USA \\ Correspondence: Ziyi Liu, Viewpoint School, 23620 Mulholland Highway, Calabasas, California 91302, USA. \\ E-mail: Z.Liu20@viewpoint.org
}

Received: November 9, 2018

Accepted: December 5, 2018 Online Published: December 30, 2018

doi:10.5539/jel.v8n1p43

URL: https://doi.org/10.5539/jel.v8n1p43

\begin{abstract}
When memorizing mechanical materials such as words or numbers, people have shown the tendency to overestimate their future memory due to their insensitivity to memory loss. The experiments in this paper investigate whether the same bias applies to conceptual learning and, if so, how the magnitude of this bias compares to that of mechanical learning. In our experiment, participants were divided into four groups. Two groups of participants studied a word list and took free-recall tests either two minutes or one week after learning; they also made predictions of how well they would do on the test right after learning the material. The other two groups were designed the same way except that the learning material was a concept. Our results indicate that the stability bias illusions not only applied to conceptual learning but were also more significant than in mechanical learning.
\end{abstract}

Keywords: metacognition, conceptual learning, mechanical learning, memory loss, stability bias, ease of processing

\section{Introduction}

Metacognition is the "cognition of cognition:" the awareness, understanding and regulating of one's own thinking. Metamemory is a specific type of metacognition - that refers to memory; more specifically, the judgments and beliefs of memory (Kornell, Rhodes, Castel, \& Tauber, 2011). Judgment is simply the prediction or evaluation of one's remembering and forgetting. In contrast, beliefs are more rational, based on one's experience and knowledge about memory. For example, "numbers are easier to memorize than words" is a belief about memory. When studying judgments of memory, researchers usually conduct experiments where the participants learn certain materials and make predictions of how much of those materials they will remember on a future date, and those predictions are what we call judgments of learning (JOLs). Various factors affect the magnitude of these judgments, and in the following experiment we will focus on one of them.

\subsection{The Stability Bias}

When asked to predict their future recalling of learned information, people tend to display two biases. First, their insensitivity to memory loss over the course of time leads them to make similar predictions for their performances on tests that would take place after different retention intervals (Carroll, Nelson, \& Kirwan, 1997; Maki \& Swett, 1987). Second, for the same reason, people usually predict better recall performance than their actual memory (Koriat \& Bjork, in press). However, the latter bias becomes nearly negligible when the study-test interval is as short and only lasts a few minutes (Koriat, Sheffer, \& Ma'ayan, 2002). These two errors-similar memory predictions for different time intervals and overconfidence in performance- constitute what we call stability bias.

Researchers have tested the effects of different variables on the extent of stability bias. For example, Kornell and colleagues (2011) conducted research using participants recruited through Amazon Mechanical Turk and word lists created based on the norms of Kucera and Francis (1967) - a corpus of 1 million English words' identified by frequency of occurrence - and concluded that changing the type size of words influenced judgments of memory but not actual memory performance. In addition, Rawson and Dunlosky (2002) discovered that high coherence of texts lead people to make falsely high JOLs. Results from these investigations yielded results that support the ease-of-processing heuristic. While the qualities of stimuli (type size, coherence, etc.) have been studied in terms of their effects on stability bias, our research will focus on a new direction: the influence of the type (conceptual versus mechanical) of material on the stability bias. 


\subsection{The Ease of Processing Heuristic}

Koriat and colleagues (2004) present a dual-basis view, which suggests that judgments of memory are based on either subjective experience during learning or priori knowledge and beliefs about memory. The former is referred to as "experience-based," the latter is called "theory-based." Theory-based JOLs are likely to be more educated and accurate because they are based on knowledge; however, they are usually activated only when learners become sensitive to memory decline by having retention-interval differences made salient to them (Koriat et al., 2004). Because this reminder of memory decline is not provided in most daily circumstances, experience-based JOLs constitute the majority of individuals' predictions of memory, and they are proven to be highly affected by the ease-of-processing of materials learned - in other words, how fluently the information can be encoded by the learner. The ease of processing brings stability bias because higher perceived ease leads to metacognitive judgments for better performance but does not influence actual memory. In conclusion, in most cases, JOLs are experienced-based, subjective, and affected by the ease-of-processing; as a result, they display stability bias. This derives the general theory that the easier it is to process information, the higher the stability bias we would expect to occur. Because conceptual materials are considered more difficult to process than mechanical information (numbers, words, etc.), we conducted an experiment comparing the stability biases in learning both materials to test if the ease of processing heuristic leads to lower stability bias in conceptual learning.

\subsection{The Current Experiment}

In this research study, we examined the magnitude of stability biases presented by participants who learned a word list and those who learned a concept that required comprehension. Although the former type of material does not require active thinking or comprehending, the latter requires analytical efforts for understanding.

Based on our theory that (1) information that requires understanding is harder to process than a list of random, individual words and (2) processing ease results in overconfidence in prediction, we hypothesize that the word list group would display greater stability bias. That is, participants who memorized words would make more similar estimates for different retention intervals, as well as generally higher JOLs for both intervals. However, we also hypothesize that word group participants display similar prediction-performance discrepancies to that of participants in the conceptual learning condition, given that words are not only easier to process but are also easier to encode and retrieve, which results in better actual recall performances. By investigating this topic, we explore whether the ease of processing heuristic applies to conceptual learning and causes it to present a smaller stability bias than that of mechanical learning. In addition, we examine whether the stability bias applies to conceptual learning at all.

In two groups, participants studied a word list and took free-recall tests on the material after either a two-minute study-test interval or a one-week interval. We recorded the JOLs for different retention intervals. The other two groups of participants studied a video explaining a concept and took free-recall tests on the concept after a two-minute or one-week interval. We again recorded the JOLs. By comparing the general magnitudes of JOLs and the differences in JOLs for different time intervals of the two material groups, we examined the first part of our hypothesis; by calculating the prediction-performance differences for both materials, we examined the second part of our hypothesis.

\section{Method}

\subsection{Participants}

A sample of 169 participants of varied genders and ages was recruited via Amazon's Mechanical Turk, a website that allows workers to complete online surveys for compensation. They were selected under the requirements of being fluent English speakers and Amazon Mechanical Turk workers with greater than or equal to $95 \%$ approval rate for all the surveys they had done. They were paid $\$ 1$ for completing tasks that took five minutes.

Thirty-nine participants were assigned to the two-minute retention interval word list condition (10 female, 29 male; range $=18-72$ years); fifty-one were assigned to the one-week word list condition ( 22 female, 29 male; range $=20-64$ years). Twenty-seven were assigned to the two-minute retention interval conceptual learning condition (14 female, 13 male; range $=22-55$ years); fifty-two were assigned to the one-week conceptual learning condition ( 22 female, 30 male; range $=21-76$ years). All participants lived in the United States and spoke English fluently.

\subsection{Materials and Procedures}

A ten-word list stimulus was generated at Paivio et al. Word List Generator. On average, the 10 words had a Kucera-Francis Frequency of 33.7, 2.4 syllables, and 7.1 letters. The words were displayed in a video that 
showed each word for 6.8 seconds. The video was 68 seconds long in total. We also provided a link to a two-minute long blank video for participants to watch as the retention interval before the test for the two-minute retention interval condition. The conceptual learning material was a video explaining a concept related to calico cats created by Carpenter and colleagues (2013). The video shows a person explaining the genetic inheritance pattern that results in the calico coat color. There were ten idea units that we used to evaluate the participants' answers. The ten idea units were also from Carpenter and colleagues (2013). The video was of the same length as the word list video, 68 seconds. For the two-minute retention interval group, we also provided a blank delay video.

After participants studied the word list, they were asked to make predictions for how many words they would be able to recall in either two minutes or one week. For people in the one-week condition, the survey ended there whereas people who were asked to predict their performance in two minutes proceeded to a video link that directed them to a two-minute long retention video. Afterwards, they typed down as many words as they could remember from the word list in a blank box. There was no time limit for the blank recall. After a week, we sent out individual letters to participants in the one-week retention interval condition for them to take a second-trial survey where they simply recalled and typed down as many words as they still remembered.

The procedure for conceptual learning groups was designed to be identical to that of mechanical learning group, with only the word list video changed to the calico cat video stimulus. Before showing them the material, we informed participants that their responses would be graded based on ten idea units, which we phrased as "essential pieces of information."

\section{Results}

All participant responses were manually graded based on how many pieces of information they got correct. Because not all participants who participated in the first session came back for session two, we only used data of the participants who completed both surveys, which is why our final data pool for analysis was smaller. We were able to analyze five participants for the two-minute word list group, eight for the one-week word list group, twelve for the two-minute concept group and nine for the one-week concept group (Table 1).

Table 1. Session one participants and returned participants

\begin{tabular}{lllllll}
\hline & \multicolumn{3}{l}{ 2-min interval } & & \multicolumn{4}{l}{ 1-week interval } \\
\hline & All & Returned & Percent Returned & All & Returned & Percent Returned \\
\hline Mechanical & 39 & 5 & $12.821 \%$ & 51 & 8 & $15.686 \%$ \\
Conceptual & 27 & 12 & $44.444 \%$ & 52 & 9 & $17.308 \%$ \\
\hline
\end{tabular}

For the word list groups, in the two-minute condition, the average JOL and actual performance scored the same number: 4.200. This result supports the idea that in studying mechanical materials, when study-test interval is minimized, predictions are often approximate to actual performances. In the one-week condition, however, participants' average JOL was 3.125 whereas their actual performance was 0.375 (Figure 1). This significant discrepancy demonstrates participants' overconfidence in their memory endurance. Finally, while the difference in JOLs for the two-time intervals was only 1.075 , their actual memory declined by 3.825 .

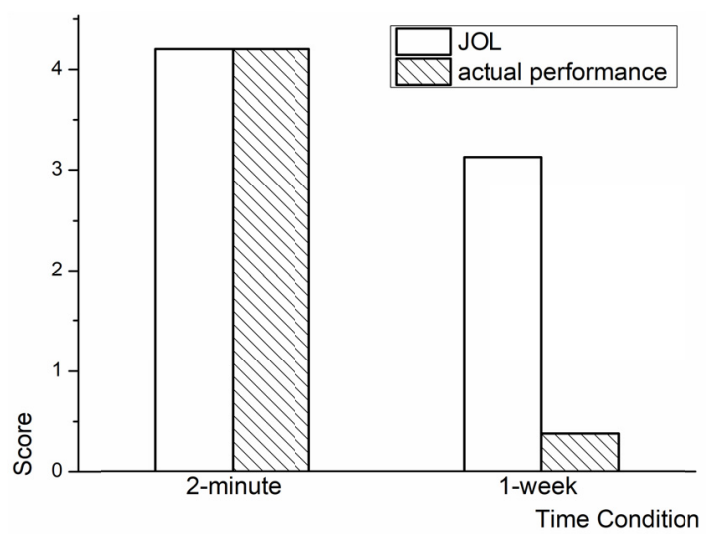

Figure 1. JOL and actual performance of the word list group 
In the two-minute condition of conceptual learning, participants predicted their recall performances to score 4.917 idea units correct out of ten idea units on average, and their actual performance average was 4.083, displaying a slight overconfidence in performance. On the other hand, the one-week group also showed overconfidence in memory endurance given their average JOL for one week later was 4.333, which was not significantly different than their predictions for the first test. Their actual performance was 1.555 , significantly lower than what they had predicted (Figure 2). Although their JOLs for the two tests differ by only 0.584 , their actual memory dropped by 2.528 . Thus, the stability bias appears to apply to conceptual learning as well as mechanical memorization, which supports our hypotheses.

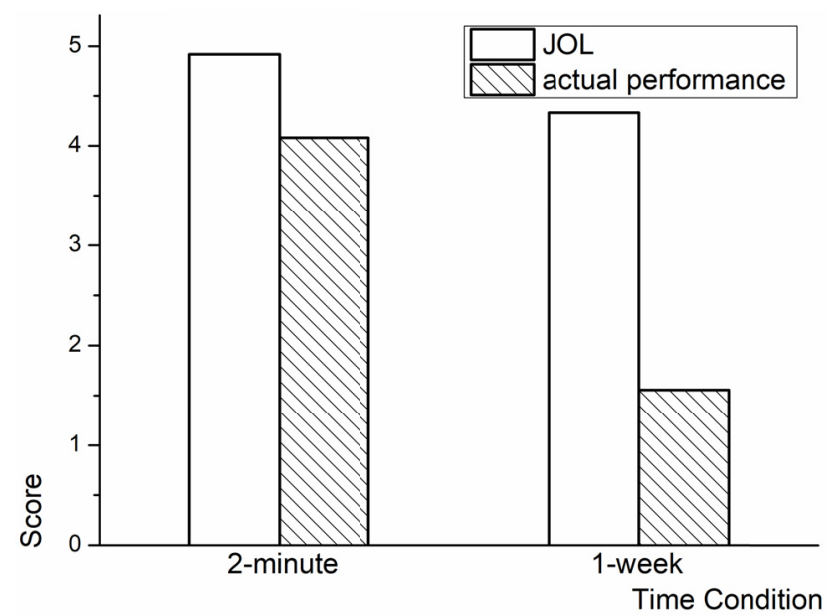

Figure 2. JOL and actual performance of the conceptual learning group

\section{Discussion}

Although we hypothesized that participants who learned words would give more similar estimations for different retention intervals and generally higher JOLs for both intervals, the results show that the conceptual learning condition actually presented a smaller difference in its two average predictions and higher JOLs. These findings suggest that people display even more overconfidence, or errors of stability bias, in conceptual learning than mechanical learning. This surprising finding conflicts with our assumption that people would be more rational when predicting memories of ideas because ideas are more difficult to process. We believe that this is because either the ease of processing heuristic does not apply to conceptual learning or because conceptual information is not in fact more difficult to process than words. Future research should examine these possibilities.

On the other hand, our results support our hypothesis that the two groups display similar prediction-performance discrepancies, especially for the one-week condition. This suggests that the predicted learning difficulties of our stimuli were directly proportional to the actual learning difficulties. Despite the word list group having 0 discrepancy for the two-minute condition and the concept group having 0.827 for the same retention interval, their discrepancies for the one-week condition were quite similar (2.750 and 2.778).

The understanding we gained from this research is that stability bias does indeed occur in conceptual learning as it does in mechanical learning. As such, metacognition errors in conceptual learning may be even more pronounced than in mechanical memorization. This finding adds to our existing knowledge regarding stability bias; we now know the fact that this bias applies to not only memorization but also comprehension, and we understand the different extents at which it applies to the two types of learning. The practical contribution of this finding is that learners can be aware of their forgetting and continuously consolidate their memory when learning concepts. Also, when learning concepts and mechanical information at the same time, people could be aware that stability bias takes place more in memorization and therefore put greater efforts into their mechanical learning.

Another finding was that despite the different magnitude of JOLs for the two different stimuli, their prediction-behavior discrepancies were similar, showing that predicted learning difficulties correspond with actual learning difficulties of materials. 


\section{Acknowledgement}

The author appreciates valuable guidance and discussions from Dr. Nate Kornell at Williams College and Travis Cramer at New York University during the research and preparation of this paper.

\section{References}

Carpenter, S. K., Wilford, M. M., \& Kornell, N. (2013). Appearances can be deceiving: instructor fluency increases perceptions of learning without increasing actual learning. Psychonomic Bulletin \& Review, 20(6). https://doi.org/10.3758/s13423-013-0442-z

Carroll, M., Nelson, T. O., \& Kirwan, A. (1997). Tradeoff of semantic relatedness and degree of overlearning: Differential effects on metamemory and on long-term retention. Acta Psychologica, 95, 239-253. https://doi.org/10.1016/S0001-6918(96)00040-6

Koriat, A., \& Bjork, R. A. (in press). Illusions of competence in monitoring one's knowledge during study. Journal of Experimental Psychology: Learning, Memory, and Cognition. https://doi.org/10.1037/0278-7393.31.2.187

Koriat, A., Bjork, R. A., Sheffer, L., \& Bar, S. K. (2004). Predicting one's own forgetting: the role of experience-based and theory-based processes. Journal of Experimental Psychology: General, 133(4), 643. https://dx.doi.org/10.1037/0096-3445.133.4.643

Koriat, A., Sheffer, L., \& Ma'ayan, H. (2002). Comparing objective and subjective learning curves: Judgments of learning exhibit increased underconfidence with practice. Journal of Experimental Psychology: General, 131, 147-162. https://dx.doi.org/10.1037/0096-3445.131.2.147

Kornell, N., Rhodes, M., Castel, A., \& Tauber, S. (2011). The Ease-of-Processing Heuristic and the Stability Bias: Dissociating Memory, Memory Beliefs, and Memory Judgments. Psychological Science, 22(6), 787-794. http://dx.doi.org/10.1177/0956797611407929

Maki, R. H., \& Swett, S. (1987). Metamemory for narrative text. Memory \& Cognition, 15, 72-83.

Paivio, A., Yuille, J. C., \& Madigan, S. A. (1968). Concreteness, imagery, and meaningfulness values for 925 nouns. Journal of Experimental Psychology, 76(1, Pt.2), 1-25. https://dx.doi.org/10.1037/h0025327

Rawson, K. A., \& Dunlosky, J. (2002). Are performance predictions for text based on ease of processing?. Journal of Experimental Psychology: Learning, Memory, and Cognition, 28(1), 69. https://dx.doi.org/10.1037/0278-7393.28.1.69

\section{Copyrights}

Copyright for this article is retained by the author, with first publication rights granted to the journal.

This is an open-access article distributed under the terms and conditions of the Creative Commons Attribution license (http://creativecommons.org/licenses/by/4.0/). 